\title{
81. A New Technical Idea for the Operation of the Intracranial Aneurysm and A-V-Malformation
}

\author{
Takayoshi NomuRA \\ Dept. of Neurosurgery, Nagoya National Hospital
}

1) Crushing of the aneurysm by silver clip.

For the sadle aneurysm, to crush the aneurysm with a few silver clips is a radical operation to retain the cerebral ciaculation, combining method with corting would be better.

2) Striping of the small branches of the arterial trunkes for a-v-malformation.

The radical operation for the a-v-malformation without typical inflow arterys are considerably difficult, because of the big damage for the brain tissue. To strip the arterial wall, from which the malformated shants get the blood flow, was resulted good effect for hide the malformation.

3) Evacuation of the subarachinoidal hematoma at the first aid of the subarachinoidal hemorrhage.

At the first aid of the subarachinoidal hemorrage, evacuation of the hematoma through the small hole of the arachinoid membrane is effective procedure to minimize the residual syndromes.

4) Decompression at the first aid of the subarachinoidal hemorrhage.

At the first aid of the subarachin oidalhemorrhage, the decompression craniotomy is very important procedure. Decompression craniotomy and evacuation of the subdural hematoma due to fuge subarachinoidal hemorrhage are quite effective. 